\title{
The Design of Teaching Experiment System Based on Virtual Instrument Technology
}

\author{
Dayong Huo \\ Department of Physics, Kashgar Teachers College, Kashgar, 844006, China \\ hnnyhdy@163.com
}

Keywords: Virtual Instrument;; LabVIEW; Teaching Experiment;Data Acquisition

\begin{abstract}
In the paper, a set of teaching experiment system based on virtual instrument technology is developed. Because the current experimental teaching system in technological college faces many important challenges, for example, the equipment gets obsolete, and the cost to construct or maintain the laboratories becomes higher, etc. Therefore, it is essential to improve the current teaching experimental system effectively. In the system, LabVIEW software serves as the development platform, and the external data acquisition is realized by DAQ card. This system can apply to the signal testing technology courses, so the researchers can simulate the signal system response, digital filters, signal generators, oscilloscopes and signal processing experiments, and the operation result can be stored, shown, or printed.
\end{abstract}

\section{Introduction}

Experiment is an extremely important tool to improve the students' practical ability and innovation capacity in engineering colleges. In recent years, the expansion of college enrollment has brought tremendous pressure to experimental teaching. Traditional practice education has been unable to meet the teaching requirements under the new situation. The current experimental teaching system in college faces many important challenges, for example, the equipment gets obsolete the cost to construct or maintain the laboratory becomes higher, and theoretical knowledge and practical teaching becomes separated. Therefore, it is urgent to look for the experimental apparatus with low cost, high performance and good scalability, which can stimulate students' interest [1].

The laboratory based on virtual instrument technology can meet the demands of various experiments with the computers and matching software. This laboratory will reduce the construction investment, while it improves laboratory utilization with the use of powerful computers. Most experiments can be realized only in the PC virtual front panel, and the students simply use the mouse to make the experimental options. Therefore, the equipment damage due to misuse of the instruments may be avoided. Furthermore, the physical impairment of the instruments can be eliminated [2].

\section{Virtual Instrument and Development Software 'LabVIEW'}

National Instruments had proposed the concept of virtual instruments at first in 1980s, and at the same time they launched the corresponding development software named LabVIEW. LabVIEW is one of the graphic programming languages written in G program, which is produced in block diagram form. The term, icons and concepts used in the program are familiar to the technical personnel, scientists and engineers as far as possible, thus the programming process becomes simple and intuitive [3].

"Software is the instrument". This idea became a reality because of the emergence of virtual instruments. The virtual instruments combined the hardware with application software through the computers. The users can operate this computer via a friendly graphical interface, just like they handle an instrument by their own definition or design. Then the virtual instruments can achieve the functions including data collection, analysis, judgment, display, storage, etc. Thus the instrument panel is virtual, and the measurement functions are realized by the software. The function of the virtual instruments 
can be divided into three modules including data acquisition, data analysis and result display, as shown in Figure 1.

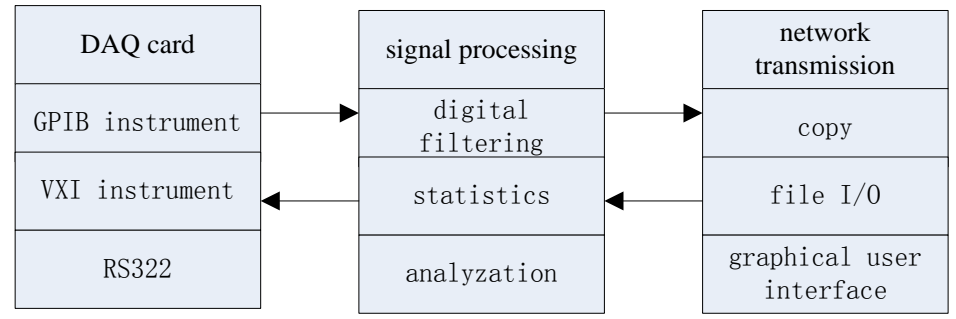

Date Collection Data Analysis and Processing Result Display

Figure. 1 Virtual Instrument Function Module Partition

\section{Overall Functional Design}

This system uses a modular idea to design three signal input modes with the software LabVIEW. The input modes include the data acquisition card hardware input mode, NI DAQ assistant analog hardware input mode, and LabVIEW signal processing mode. The software can analyze the input signals, and the output result is indicated on the front panel in data or graphical form. The main function of the system is illustrated in Figure 2.

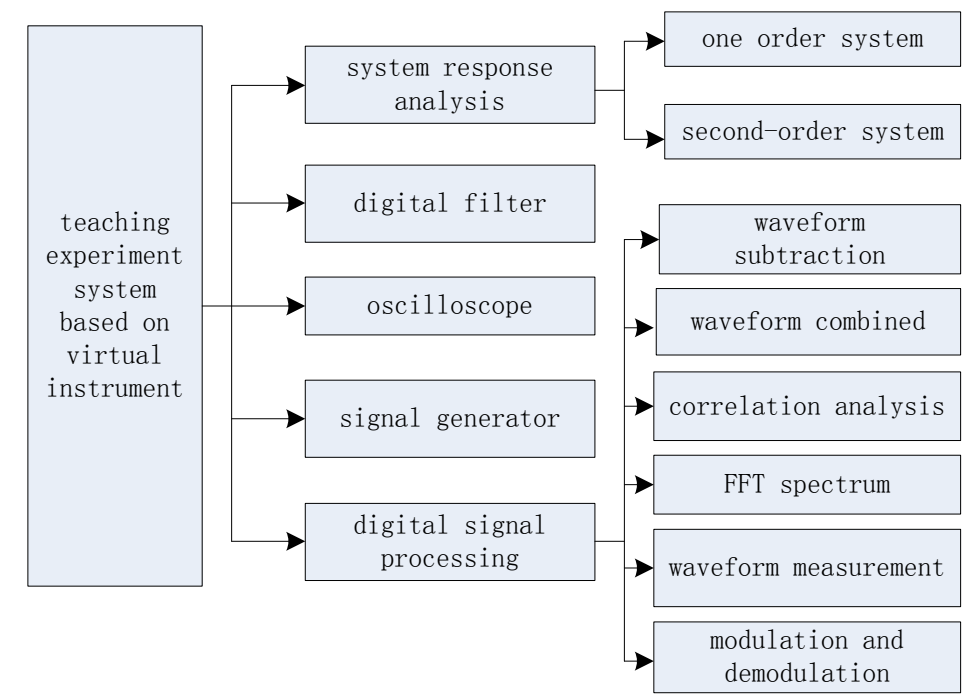

Figure. 2 The Whole System Functions Framework

The main features of the system include the following five categories: response analysis, digital filters, oscilloscopes, signal generators and digital signal processing function. In the module of system response analysis, the paper can complete the first-order and second-order system response analysis. In the digital signal processing module, the paper can complete the multi-channel input signal analysis, such as signal spectrum analysis, correlation analysis, modulation and demodulation, etc. Each module has been designed with data storage capacity in order to facilitate further processing of data, and then the paper can save the result in the form of graphics or data [4].

\section{Composition of Virtual Experiment System}

The system comprises of software and hardware components. The hardware includes the data acquisition card, external input, and PC machines. The PCI data acquisition card is the core component of the system 
hardware, which is used to transfer and process the data of external input. The main part of the software is based on LabVIEW 8.6 to develop the necessary procedures.

Hardware configuration. The system utilizes a PCI-1802H multifunction data acquisition card, which can be plugged into the computer's PCI slots directly. The performance of the card affects the running accuracy and timeliness of the whole system. The PCI-1802H card can achieve a smooth continuous data acquisition $330 \mathrm{KHz}$ under DOS and windows environment. It includes two 12-bit D / A output channels, 16 digital input channels and 16 digital output channels. Meanwhile, the PCI-1802H card offers 32 single-ended or 16 differential inputs.

The 'Magic Scan' function is an innovative design in this DAQ card. Under the function, the system can scan the different input channels at a huge difference rate, and its gain is different. Even when the system scans the multi-channels, the sampling rate can be maintained at 330KS / s.

Software constitutes. Through the use of LabVIEW 8.6, the software system can drive data acquisition card. In addition, the software system can accomplish the input signal analysis, processing, storage, and complete the system interface design.

The system has three optional modes to input signals. External hardware input mode is offered to the students. External hardware can be a variety of sensor signals or student-produced circuit signals. For example, the circuit signals designed by the students can be verified and displayed in the oscilloscope of the system, and the corresponding signal analysis can be proceeded simultaneously. The signals can be collected by the data acquisition card through the I/O channels, then they are inputed into the system after the $\mathrm{A} / \mathrm{D}$ or D/A conversion. The flow of data acquisition is illustrated in Figure 3. The data acquisition card can work in various drive modes. In this system, LabVIEW provides call library functions (CLF). Then the paper can use the dynamic link library to drive the data acquisition card [5].

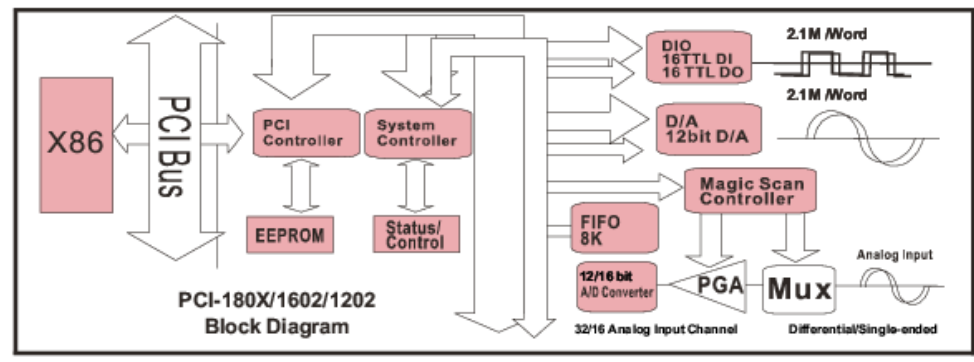

Figure 3 The Flow of Data Acquisition

If you do not have the data acquisition card, you can use NI-DAQ to simulate the desired voltage or current signal input as generated by the hardware. NI-DAQ can simulate various types of data acquisition card, and set the parameters of the analog channels with the LabVIEW software.

Because LabVIEW has a powerful signal analysis function, the other signal sources in this system are the signal module of the software.

The main interface of the system is convenient for the students to apply. LabVIEW can bring together the various sub-functions of the system to the main interface for teachers and students to choose.

\section{Application Examples}

This example is an experiment with signal processing functions. The experiment can analyze the single or dual channel input signals, and students can choose as needed.

The signal processing module can display and analyze each input signals. Compared with the traditional oscilloscope or signal analyzers, the module has many advantages of low price, multifunction, programmability and visual display, etc. This system can display the collected signal in real time through "Waveform Graphs", then save the waveform as LVM file with "Write To Measurement File". Therefore, the basic "store" function is realized. Vice versa, the system can read the display via "Read To Measurement File LVM", thus complete the "echo" function. 
If the system is built on the PC and capture card, the oscilloscope may have some restrictions on the sampling rate, bandwidth, resolution and other parameters. The response time of the procedures depends on the PC configurations. The main functions are listed as follows.

- Real-time Display: The input signals can be collected by the signal acquisition card, and displayed on a PC terminal in real time.

- Digital Filter: This digital filter is used to process the signals and display the result in real time. Arbitrarily we can set all kinds of parameters including the best approximation function type, the filter type and order, upper and lower cut-off frequency, etc.

- Waveform Display: The system can realize the transient waveform display, and you can save the transient waveform.

- Waveform Storage: At any time we may store the original signals or the processed signals on the local hard disk in LVM file form, which is unique in the LabVIEW. The transient signal can be saved on the local hard disk in BMP image formats for later viewing or analysis.

- Waveform Reappearance: The waveform files stored in LVM format can be read at any time, then displayed on the PC side.

- Spectrum Analysis: After the filter process of the signals, the amplitude-frequency response analysis and phase-frequency response analysis are performed, and the real-time waveform display is realized.

\section{Conclusion}

The paper has established a virtual instrument experimental teaching system, which facilitates the students' experimental operation on the same computer interface. Given the enormous data computing and signal processing functions of the computer with LabVIEW software, the paper can also connect the system to other software, such as MATLAB, VC + +, etc. Compared to the conventional laboratory equipments, the system can save instrument hardware costs significantly. At the same time, the system is convenient for the operator to process further development, which provides a new method to develop students' practical ability, design ability and creativity.

\section{References}

[1] Ryan A Sherry, Susan M. Lord. Lab VIEW as an Effective Enhancement to an Optoelectronics

[2] Laboratory Experiment. IEEE Transactions on Circuits and System, 1997, Page(s): 904-907.

[3] Edward M. O'Brien, Aaron s. Collins, William M, Moses Measurement of Thermocouple Time Constants Using LabVIEW As Part of an Engineering Recruiting Weekend for high School Seniors. IEEE DocumentAnalysis and Recognition. 2001, P. 122.

[4] Engr.Atif Ahmed Siddiqui, Engr.M. Yousuf Irfan zia, Engr.Muhammed Aamir, Virtual Remote Electronic Laboratory, IEEE Transactions on Signal Processing, 2002, p. 691.

[5] National Instrument. LabVIEW Signal Processing Course Manual.1997 (9).

[6] Hong Lai, Yinfeng Wang: Research of Reconfigurable Virtual Instrument Technology, Advanced Materials Research, Vol. 711 (2013), p. 571.

[7] Zhou Yang. Research on Automatic Test system for Mixed-Signal Circuits Based on Virtual Instrument Technology. JILIN University, China, 2013, pp. 20-35.

[8] QIAO Yu-jing, WEI Jian-yu, XIE DI. Development of Experiment Platform for Testing Technology Based on Virtual Instrument, Techniques of Automation \& Applications. 31(2012)75-77. 
[9] You Lihua, Zhou Yang, Building of Testing Technology Experimental Teaching System based on Virtual Instrument, Experimental Technology and Management.28(2011)83-86.

[10]LI Xue-cong, WANG Pin, GU Gang, Innovativ Experimental Teaching Platform in Measuring Technology Based on Virtual Instrument, Journal of Guangdong University of Technology. 8(2008)140-141.

[11]TAN Huang, LIU Yi, ZHENG Xue-ren, The Teaching System of Electronic Experimment Based on Virtual Instrument,Control \&Automation.84(2008)86-87 